\title{
Essai de vaccination antibovipestique de veaux passivement immuns par anticorps d'origine colostrale avec un vaccin inactivé adjuvé préparé en cultures cellulaires
}

\author{
par A. PROVOST, C. BORREDON et Y. MAURICE* \\ (I. E. M.V.T., Laboratoire de Recherches Vétérinaires de Farcha, Fort-Lamy, Tchad)
}

\section{RÉSUMÉ}

L'inoculation d'un vaccin de culture cellulaire du virus bovipestıque ınactıvé par la ß-propiolactone et adjuvé por l'adjuvant A 65 n'apporte aucune protection, dans les mols suivants, aux veaux qui le reçoivent alors qu'ils hébergent encore des anticorps antipestiques d'origine maternelle. Le problème de la vaccination antibovipestique des vecux reste posé dans les campagnes de vaccination.

Le planificateur de campagnes de vaccination antipestique a de sérieux problèmes techniques à résoudre; témoin en est la campagne interafricaine connue sous le sigle P. C. $15(11,12)$. Le problème de la vaccination en pays d'enzootıe pestique des veaux nés de vaches immunes est l'un d'eux. II n'y a pas lieu de le présenter de nouveau: les bases scientifiques ont été mises en lumière par POLSON (14) et BROWN (4), les limites de l'applicabılıté des vaccins caprinisés ou de cultures cellulaires ont été étudiées par STRICKLAND (20) et SMITH (21) et le bien-fondé de ces observations reconnu par LÉPISSIER et MACFARLANE (11) à la fin des opératıons vaccinales de la première phase du PC 15. Le conseil, alors donné, de faire repasser plusieurs fois les équipes de vaccination pour vacciner les

(*) Notre très vive gratitude est acquise à Monsieur le Contrôleur d'Elevage Jean FADÉ qui a assuré la surveillance des veaux d'expérience dans des conditions pralıques difficiles. veaux à un âge adéquat paraissait pourtant être de réalisation difficile dans certains états comme le Tchad, pays de communication difficiles et où l'impératif de la transhumance rend pratiquement illusoire l'espoir de retrouver les veaux à revacciner.

Parfaltement conscient de la pierre d'achoppement que représentalt ce problème de la vaccination des veaux, problème d'autant plus aigu en région sahélienne que les tournées de vaccination sont annuelles et qu'en conséquence c'est le croît annuel du cheptel bovin qui se trouve chaque année exposé au contage, le Laboratoire de Farcha s'est depuis plusieurs années penché sur sa résolution. Une première solution a été apportée par la démonstration de l'efficacité de la souche MB 113 Y du virus de la rougeole pour la protection hétérologue des veaux (15). Ce virus peut être inoculé avec succès à un âge où le vaccin de cultures cellulaires est inefficace par suite du relıquat d'anticorps d'origine colostrale; par ailleurs, il induit chez 
la majorité des veaux qui l'ont reçu des anticorps antipestiques neutralisants de titre comparable à celui de la vaccination antipestique homologue, tandis que ceux quı n'ont pas répondu restent justıciables de cette dernière vaccination. L'ımportance du «hiatus Immunitaıre de l'âge», période pendant laquelle les veaux sont de nouveau sensibles à la peste, est donc réduite maıs non totalement annulée pulsque certaıns veaux, ceux dont le titre en anticorps antipestiques colostraux se situe entre $\mathrm{TN}_{50}=1$ à $1,2 \mathrm{au}$ moment de l'inoculation de la souche MB 113 Y, neutralısent le virus inoculé et se retrouvent sensibles après avoir perdu ces anticorps d'origine maternelle. La solution n'est donc pas entièrement satisfaisante. Une autre voie d'abord pourrait être l'emploi d'un vaccin inactivé inoculé aux vecux passivement immuns. Sı un tel vacan se révélat être Immunigène chez eux, l'immunité engendrée - on doit s'y attendre par ce que l'on salt de ce type de vaccin (17) - ne devrait être que de quelques mols, au plus deux ans. Dans le cas présent, il fallait qu'elle solt suffisamment longue pour permettre aux veaux perdant leur immunité passive transmise de résister au contage éventuel avec le bénéfice de l'immunité active qu'ils auraient élaborée, mais aussi suffisamment brève ou de qualité telle qu'ils puissent réagir avec succès d̀ la revaccination l'année suivante. Un travail déjà ancien, de JACOTOT et COLSON (7 a) laissait à penser que la méthode était valable, d'autant que DAUBNEY (4a) en avalt lui aussi été satisfaiten conférant à des veaux temporairement immuns une immunité active de plus de 12 mois.

C'est sur ces bases qu'il fut décidé d'entreprendre l'expérience, objet de ces lignes. Le vaccin sous test est un vaccin expérimental inactivé et adjuvé, préparé avec un virus de cułtures cellulaires et qui, dans des essas préliminaires, s'était révélé d'une efficacité móyenne $(8,9)$; Il paraissait être indiqué pour ce qu'on demandait de lui dans l'expérience.

\section{MATÉRIEL ET MÉTHODES}

1. - Plan d'expérience. Défini d'accord parties avec le Service de I'Elevage du Tchad, l'expérience a pour buts :

- de montrer combien de temps l'inoculation d'un vaccın antıpestıque inactivé protège les veaux pendant la période où Ils recouvrent leur réceptivité au contage pestique (entre 6 mois ef 1 an), époque pendant laquelle aucune vaccinatıon antipestique n'est pratiquée puisque cette opération s'effectue normalement d'octobre à mars.

- corollaire de ce qui vient d'être dit, montrer qu'ils peuvent être revaccinés à l'âge de 14-18 mois avec un vaccin antipestique de cultures cellulaires sans que la vaccination antérieure au vaccon inactivé entrave l'immunogenèse due au vaccin vivant.

Par suite d'une sıtuation socio-politique particulière interdisant les déplacements en brousse, seule la première partie du plan a pu être menée à bien.

2. - Vaccin inactivité adjuvé de cultures cellulaires. Le protocole de préparation décrit en 1965 (8) व été modifié en certains points, notamment en ce qui concerne l'adjuvant de type Freund alors utilisé et qui donnait des réactions locales qui auraient été inacceptables chez des vequx.

On obtient des couches monocellulaires de cellules de rein d'embryon de veau en seconde explantation par les procédés classiques de cultures cellulaires.

Elles sont infectées avec la souche bovipestique pathogène DK sous forme d'un broyat de rate au $1 / 10$ lyophilisé. On suit tous les jours la progression de l'effet cytopathique ef I'on attend qu'il soit total, ce qui se réalise en 6 à 8 jours, point différent de la méthodologie de production des vaccins vivants de cultures cellularres; on s'attend, ce faisant, à ce que les liquides de cultures récèlent une importante population de virions pestıques thermo-inactivés.

Les liquides de cultures cellulaires sont clarifiés par filtration dans le seul but de retenır les débris cellulaires, puis refroldis à $4{ }^{\circ} \mathrm{C}$ sous agitation magnétique; on ajoute alors 0,1 p. 100 $(v / v)$ de $\beta$-propiolactone. On contınue à agıter

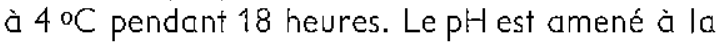
neutralıté avec quelques gouttes de soude centinormale lorsqu'une prise d'essal montre un abaıssement vers 6,5. L'inactivation du virus est contrôlée par ensemencement sur cellules de rein d'embryon de veau qui restent normales pendant 15 jours d'observation. 
Le vaccin est finalement réalisé en mélangeant à parties égales sous l'agitation d'un ultraTurax* le liquide inactivé et l'adjuvant A65 (22). Préconisé en médecine humaine, de valeur égale à celle de l'adjuvant de FREUND (6), c'est un produit métabolisable constitué de 86 p. 100 d'huile d'arachide, 10 p. 100 d'Arlacel et 4 p. 100 de monostéarate d'aluminium ; sa préparation est aisée sous mixage.

Le produit final est rose nacré, de consistance sirupeuse, passant bien dans la seringue. L'émulsion reste stable au réfrigérateur, où elle sédimente sans se détruire; elle reprend son homogénéité par simple agitation manuelle.

3. - Veoux d'expérience. L'expérience a eu lieu à Bokoro (Tchad) sur des veaux mis à notre disposition par leurs propriétaires. Tablant sur la confiance et le bon vouloir de ces derniers, il fallait que les opérations soient les moins gênantes pour eux. Le choix de l'emplacement était par ailleurs dicté par l'assurance que l'on avait de retrouver les animaux tout au long de l'année, abreuvement ef affouragement étant localement en suffisance.

Deux cents veaux, âgés pour la plupart de 2 à 7 mois et ne portant aucune marque auriculaire de vaccination, entrent en expérience. Celle-ci ayant débuté à la fin de la première phase du PC 15, on peut penser que toutes les mères ont été vaccinées contre la peste au moins une fois dans des conditions valables d'immunisation, ce que révèle un sondage sérologique pratiqué sur 25 d'entre elles fournissant 25 réponses positives.

Cent veaux (nos 1201 à 1300) reçoivent par voie sous-cutanée dans le pli du fanon $2 \mathrm{ml}$ de vaccin inactıvé après une prise de sang destınée d̀ contrôler leurs anticorps. Quatre-vingt-dix autres veaux ( $n^{\text {os }} 1831$ à 2020) reçoivent au même endroit un placebo d'eau distıllée et dix autres (nos 1821 à 1830) une dose vaccinale de vaccın caprinisé. Les températures rectales de ce dernier groupe sont prises pendant 10 jours ; il est le témoin de la réactivité de l'ensemble des veaux inoculés.

Deux mois plus tard, on fait une prise de sang à tous les animaux de l'expérience, puis 10 veaux vaccinés et 10 veaux ayant reçu le placebo sont

(*) Labo. Moderne, 6, rue de la Vrillère, Paris. éprouvés avec le vaccin caprinisé ; les températures rectales sont prises pendant 10 jours pour comparer la réactivité des deux groupes. La même opération est renouvelée 2 mois plus tard, soit 4 mois après l'opération initiale. Prévue pour durer 1 an sur le même rythme, l'expérience a dû être arrêtée sine die pour des motifs indépendants de notre volonté et de celle des éleveurs et qu'il n'y a pas lieu de détallier ici. Fait heureux, on le verra, les conclusions auxquelles on a pu aboutir sont néanmoins valables.

4. - Technique sérologique. On a employé l'inhibition de l'hémagglutination morbilleuse (2) dans le seul but de contrôler une éventuelle conversion sérologique des veaux lors de leur épreuve capripestique.

\section{RÉSULTATS}

Les réactions locales à l'endroit de l'ınoculation ont été modérées et n'ont amené aucune protestation des propriétaires. La vaccination $n$ 'a entraîné de conversion sérologique, mesurée par l'inhibition de l'hémagglutınation morbilleuse, que chez un seul veau ( $\left.n^{\circ} 1224\right)$.

L'ensemble des résultats des épreuves avec le virus capripestique, révélatrice de la réceptivité ou au contraire de l'immunıté passive ou active, est colligé dans le tableau no 1.

Le statut immunitaire de l'ensemble des veaux est fourni par l'épreuve du jour J de la vaccinatıon. Cinq veaux sur 10 du groupe-témoin, d'âge moyen 5,7 mois, ont réagi. Pourtant le comportement du veau no 1823 est parfaitement aberrant : âgé de 12 mois, Il auralt dô réagir ou élaborer des anticorps ; il est vraisemblable qu'il avait été vacané et non marqué dans les opérations du B. C. 15. Dans ces conditions, il seralt justifié de l'exclure des calculs. On trouve alors que 2 veaux sur 9 sont parfaitement sensibles et 2 autres partiellement. La protection du groupe par anticorps résiduels est alors de 44 p. 100 pour un àge moyen de 5 mols.

Intéressant est le comportement des deux groupes, l'un vacciné, l'autre non, à l'épreuve faite 2 mois après la vaccination. Dans le groupe témoln, on ne relève qu'un seul veau ( $n^{\circ} 1856$ ), alors âgé de 5 mois, ne réagissant pas thermiquement ou n'élaborant pas d'anticorps. La protec- 
TABLEAU No 1

Comportement clınique et sérologique de 2 groupes de vequx, l'un vacciné avéc le vaccin inactivé-adjuvé et l'autre non vaccıné, lors d'épreuves avec le virus capripestique

\begin{tabular}{|c|c|c|c|c|c|c|c|c|}
\hline \multirow{2}{*}{ Groupe } & \multirow{2}{*}{ Numéros } & \multirow{2}{*}{$\begin{array}{c}\text { Age } \\
\text { (mois) }\end{array}$} & \multicolumn{2}{|c|}{ Jour vaccination } & \multicolumn{2}{|c|}{\lrcorner+2 mois } & \multicolumn{2}{|c|}{$\mathrm{J}+4$ mois } \\
\hline & & & IHM & $\begin{array}{c}\text { Epreuve } \\
\text { VCP }\end{array}$ & IHM & $\begin{array}{l}\text { Epreuve } \\
\text { VCP }\end{array}$ & $\mathrm{IHM}$ & $\begin{array}{l}\text { Epreuve } \\
\text { VCP }\end{array}$ \\
\hline 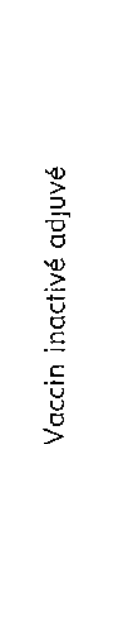 & $\begin{array}{l}1203 \\
1204 \\
1205 \\
1207 \\
1208 \\
1217 \\
1219 \\
1220 \\
1224 \\
1226 \\
1227 \\
1228 \\
1231 \\
1232 \\
1235 \\
1236 \\
1237 \\
1247 \\
1253 \\
1276\end{array}$ & $\begin{array}{r}4 \\
6 \\
5 \\
7 \\
1 \\
4 \\
5 \\
5 \\
6 \\
4 \\
4 \\
6 \\
1 \\
1 \\
6 \\
4 \\
1 \\
7 \\
12 \\
3\end{array}$ & $\begin{array}{l}<2 \\
<2 \\
<2 \\
<2 \\
<2 \\
<2 \\
<2 \\
<2 \\
<2 \\
<2 \\
\mathrm{NF} \\
\mathrm{Tr} \\
<2 \\
<2 \\
<2 \\
<2 \\
<2 \\
\mathrm{Tr} \\
<2 \\
<2 \\
<2\end{array}$ & & $\begin{array}{l}<2 \\
<2 \\
<2 \\
<2 \\
<2 \\
<2 \\
<2 \\
<2 \\
<2 \\
<2 \\
<2 \\
<2 \\
<2 \\
<2 \\
<2 \\
<2 \\
<2 \\
<2 \\
<2 \\
<2 \\
<2\end{array}$ & $\begin{array}{l}R \pm \\
R \pm \\
R- \\
R \pm \\
R- \\
R- \\
R- \\
R+ \\
R \pm \\
R-\end{array}$ & $\begin{array}{r}<2 \\
<2 \\
32 \\
32 \\
<2 \\
N F \\
N F \\
H \\
<2 \\
\dagger \\
N F \\
\dagger \\
N^{+} \\
N F \\
N F \\
<2 \\
32 \\
<2 \\
N F \\
<2\end{array}$ & $\begin{array}{l}R \pm \\
R+ \\
R+ \\
R+ \\
R+ \\
R \pm \\
R \pm\end{array}$ \\
\hline 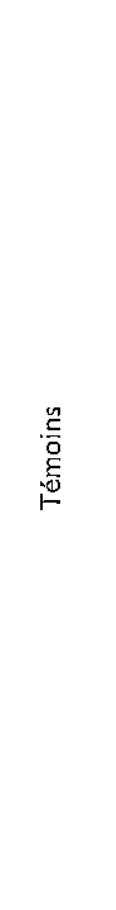 & $\begin{array}{l}1821 \\
1822 \\
1823 \\
1824 \\
1825 \\
1826 \\
1827 \\
1828 \\
1829 \\
1830 \\
1836 \\
1837 \\
1838 \\
1841 \\
1842 \\
1847 \\
1853 \\
1854 \\
1855 \\
1856 \\
1857 \\
1863 \\
1869 \\
1871 \\
1873 \\
1874 \\
1875 \\
1876 \\
1877 \\
1878\end{array}$ & $\begin{array}{r}8 \\
5 \\
12 \\
7 \\
8 \\
3 \\
3 \\
3 \\
4 \\
4 \\
6 \\
7 \\
4 \\
6 \\
3 \\
2 \\
12 \\
3 \\
5 \\
3 \\
4 \\
4 \\
1 \\
1 \\
3 \\
3\end{array}$ & $\begin{array}{l}<2 \\
<2 \\
<2 \\
<2 \\
<2 \\
<2 \\
<2 \\
<2 \\
<2 \\
<2\end{array}$ & $\begin{array}{l}R+ \\
R \pm \\
R \pm \\
R+ \\
R+ \\
R \pm \\
R+ \\
R \pm \\
R+ \\
R \pm\end{array}$ & $\begin{array}{r}2 \\
<2 \\
2 \\
\dagger \\
<2 \\
8 \\
<2 \\
2 \\
N F \\
N F \\
<2 \\
N F \\
<2 \\
<2 \\
\\
N F \\
<2 \\
<2 \\
N F \\
N F \\
<2\end{array}$ & $\begin{array}{l}\mathrm{R} \pm \\
\mathrm{R} \pm \\
\mathrm{R} \pm \\
\mathrm{R} \pm \\
\mathrm{R} \pm \\
\mathrm{R}= \\
\mathrm{R}= \\
\mathrm{R}= \\
\mathrm{R} \pm \\
\mathrm{R} \pm\end{array}$ & $\begin{array}{r}4 \\
8 \\
16 \\
4 \\
16 \\
N F \\
<2 \\
4 \\
8 \\
\dagger^{8} \\
4 \\
4\end{array}$ & $\begin{array}{l} \\
R+ \\
R+ \\
R+ \\
R+ \\
R+ \\
R+ \\
R \pm \\
R+\end{array}$ \\
\hline
\end{tabular}

Les titres des anticorps inhibant l'hémagglutination marbilleuse (IHM) sont donnés par l'inverse de la dilutıon inhıbitrice.

$\mathrm{R}+$ : réaction thermıque à l'ınoculation de virus capripestique (VCP).

$R \pm$ : réaction très modérée.

$\mathrm{R}=$ : absence de réaction.

NF : réaction sérologique non exécutée.

$\uparrow \quad$ : animal disparu ou mort. 
tion du groupe tombe à 10 p. 100 , avec un âge moyen de 5, 6 mois. Le groupe vacciné, pour son compte, possède 5 veaux qui répondent par de la fièvre ou une montée d'anticorps et un veau qui réagit d'une manière équivoque. La protection est de 40 p. 100 pour un âge moyen de 5, 6 mois, comparable à celui du groupe témoın.

Cet heureux état de choses apparent, où l'on est tenté de voir !'influence bénéfique de la vaccination, n'existe plus à l'épreuve du $4 \mathrm{e}$ mois. Dans le groupe témoin alors âgé en moyenne de 6, 9 mois, 8 veaux réagissent; 2 veaux ( $\mathrm{n}^{\mathrm{os}} 1873$ et 1877), respectivement agés de 7 et 6 mois, résıstent à l'épreuve ; la protection clinique est de 20 p. 100 . On peut la juger pour nulle dans le groupe vacciné. L'influence de la vaccination, si elle existe, n'est là que pour donner des réactions thermıques bâtardes.

Une plus juste appréciation de l'efficacité de la vaccination peut être donnée en regroupant les veaux par classes d'âge au moment des épreuves par virus capripestique; le tableau $n^{0} 2$ tente de le faire.

La protection de 20 p. 100 du groupe vacciné n'est pas significative au regard de celle de 17 p. 100 du groupe témoin. Pourtant on pourrait avoir l'impression que dans la tranche d'âge de 6-7 mois, la vaccination a été partiellement efficace (50 p. 100 pour le groupe vacciné : $22,5 \mathrm{p}, 100$ pour le groupe témoin).

Les chiffres sont trompeurs et il faut tenir compte de l'immunité colostrale résiduelle qui se fait sentir jusqu'au $7 \mathrm{e}$ mois. Ansi les pourcentages de protection dans la tranche d'âge de 3 à 7 mois s'établissent respectivement à 40 p. 100 pour le groupe vacciné et $25 \mathrm{p}$. 100 pour le groupe témoin. Mals l'apparente efficacité de la vaccination semble tomber vite, puisque, si l'on considère la tranche d'âge de 3 à 9 mois, les taux de protection ne sont plus respectivement que de 26,6 et 25 p. 100 ; si immunisation active il y a eu de par le vaccin, elle est éphémère. De plus en consultant le tableau 2, il est manifeste qu'à partir du $7 \mathrm{e}$ mois de leur vie, vaccinés ou non, tous les veaux réagissent. La conclusion est nette pour le but recherché : la vaccination avec un vaccin inactivé ef adjuvé n'est d'aucune utilité pour couvrir le «hiatus immunitaire» des veaux recouvrant leur réceptivité à la peste après avoir perdu leurs anticorps d'origine maternelle.
TABLEAU Na 2

Regroupement des veaux vaccinés ou témolns, suivant leur âge lors des épreuves par le virus capripestique

\begin{tabular}{|c|c|c|c|c|c|c|}
\hline \multirow[b]{2}{*}{$\begin{array}{c}\text { Age } \\
\text { en } \\
\text { mois }\end{array}$} & \multicolumn{3}{|c|}{ Vaccinés } & \multicolumn{3}{|c|}{ Témoins } \\
\hline & $\begin{array}{l}\text { Réac- } \\
\text { tion } \\
\text { posi- } \\
\text { tive }\end{array}$ & $\begin{array}{l}\text { Réac- } \\
\text { tion } \\
\text { néga- } \\
\text { tive }\end{array}$ & p. 100 & $\begin{array}{l}\text { Réac- } \\
\text { tıon } \\
\text { posi- } \\
\text { tive }\end{array}$ & $\begin{array}{l}\text { Réac- } \\
\text { tion } \\
\text { néga- } \\
\text { tive }\end{array}$ & p. 100 \\
\hline $\begin{array}{r}3 \\
4 \\
5 \\
6 \\
7 \\
8 \\
9 \\
10 \\
11 \\
\geqslant 12\end{array}$ & $\begin{array}{l}2 \\
1 \\
1 \\
2 \\
3 \\
2 \\
3 \\
1 \\
1\end{array}$ & $\begin{array}{l}2 \\
1\end{array}$ & $\begin{array}{r}33 \\
0 \\
06 \\
33 \\
0 \\
0 \\
0 \\
0 \\
0\end{array}$ & $\begin{array}{l}1 \\
2 \\
5 \\
4 \\
3 \\
4 \\
1\end{array}$ & $\begin{array}{l}2 \\
1 \\
1 \\
1\end{array}$ & $\begin{array}{c}66 \\
0 \\
16,6 \\
20 \\
25 \\
0 \\
0\end{array}$ \\
\hline & 16 & 4 & 20 & 24 & 5 & 17,2 \\
\hline
\end{tabular}

P. $100 \mathrm{P}$ : pourcentage de protection.

\section{DISCUSSION}

Le vaccin présenté dans ces lignes est d'un type nouveau par la source de virus, par le procédé d'inactivation et par l'adjuvant ajouté, Des essais avaient été réalisés au Kénya en 1956-57 par SCOTT ef WITCOMB (17) qui utilısaient des liquides de cultures cellulaires du virus bovipestique, inactivés par le formol ou le rayonnement ultraviolet et mélangés à de l'hydroxyde d'alumınıum. On ne possède pas de détails sur l'efficacité de ce vaccin expérimental (16). Dans les essais rapportés ici, la ß-propiolactone a été substituée au formol. La concentration de 0,3 p. 100 préconisée par STONE et DELAY (19) a été abaissée à 0,1 p. 100 pour nuire le moins possible aux antıgènes viraux; elle se montre parfaitement suffisante pour inactiver le virus. L'emploi de l'adjuvant A65 est nouveau en médecine vétérinaire. Il a été montré por l'équipe de HILLEMAN $(6,22)$ que sa valeur adjuvante était celle de l'adjuvant de FREUND, résultat confirmé au laboratoire de Farcha avec le vaccin antipestique inactivéadjuvé lui-même $(B, 9)$.

Ce n'est pas, en effet, la première fois que ce vaccin est utilisé expérimentalement. Des essais menés pendan 18 mois ont montré qu'il assurait une protection antipestique clınique valable 
pendant 2 mois, puis partielle - empêchant les bovins non de s'infecter, mais de succomber à la peste - pendant les 10 mois suivants. Il ne s'agit donc pas d'un vaccin extraordinaire et c'est la raison pour laquelie il n'a pas quitté le stade du laboratoire hormıs la présente expérience.

La critique qui pouvait venir à l'esprit quant d̀ la qualité ımmunigène intrınsèque douteuse du vaccin est ainsi réfutée par ce qui vient d'être exposé puisque le lot utilisé a lui-même été contrôlé. II faut pourtant reconnaître, on vient de le dire, que c'est un immunigène médiocre mais, gardant à l'esprit l'argumentation présentée dans l'introduction, son défaut pouvait devenir une qualité. II n'en a rien été chez les veaux.

L'expérience qui vient d'être rapportée n'est au demeurant pas la première du genre. On trouve quelques lignes dans un rapport de BRANAGAN et HAMMOND (3) sur une tentative d'immunisation de veaux avec un vaccin inactivé; elle s'est soldée elle aussi par un échec, les vaccinés contractant la peste lors du passage de la vague épizootique, mettant en défaut les opinions déjà citées de JACOTOT et COL$\operatorname{SON}(7 \mathrm{a})$ et de DAUBNEY (4a).

Le principe immunologique de la vaccination des veaux passivement immuns par anticorps colostraux a de fortes analogies avec la sérovaccination antipestique, préconssée autrefois en Asie et en Afrique. C'est JACOTOT qu। paraît avoır essayé le premier le procédé (7), suivi par BERGEON et CẺBE (1) qui enregistrent de bons résultats. Il a par contre donné des déboires à LALANNE en Côte-d'lvoire (10) et à VALLÉE, PHILIPPE et BOURDIE (21) qui concluent à l'action antagoniste du sérum et du vaccin formolé.

Le fait est bien connu en Immunologie comparée et n'a rien pour surprendre; il est établi en virologie où l'on connaît les difficultés du dosage exact du sérum et du vaccın antirabique pour que l'un interfère le moins possible sur l'autre.

En ce qui concerne la vaccination des jeunes sujets immuns et restant uniquement dans le groupe des myxovirus avec des vaccins inactivés, des échecs ontété enregistrés par OTT, GORHAM ef FARREL (13) avec de jeunes furets. Dans la rougeole, ENDERS-RUCKLE(5) a elle aussi montré que les anticorps maternels résiduels gênaient chez l'enfant l'immunisation morbilleuse avec le vaccin inactivé au tween-éther.

Cette ligne de recherches, au total, n'est pas spécialement encourageante pour le problème posé, celui de l'immunisation des veaux. L'immunisation morbilleuse présentant elle aussi des objections théoriques (transfert passif d'anticorps homologues morbilleux à la génération suivante) en cours de vérification, il paraît difficile de préconiser une solution simple. La plus logique pour l'instant, mais aussi la moins aisée et la plus coûteuse, est de farre passer plusieurs fois les équipes vaccinales. On accordera qu'il y a certes des progrès à accomplir mais que, replacée dans le contexte de la pathologie comparée, la situation est la même dans toutes les espèces pour l'immunisation active des jeunes.

\section{SUMMARY}

Rinderpest vaccination trial of colostrum immune calves with a cell-cultured inactivated and adjuvated vaccine.

Calves still harbouring colostrum-transmitted rinderpest antibodies at the time of vaccination are nol protected in the following months by a cell-cultured, $\beta$-propiolactone inactivated, A 65 adjuvated vaccine. The problem of calf vaccination in rinderpest campaigns remains unsolved.

\section{RESUMEN}

Ensayo de vacunación coníra la peste bovina de terneros pasiyamente inmunes por anticuerpos originarios del calostro con una vacuna inactivada adyuvada, preparada en cultivos celulares

La inoculación de una vacuna de cultivo celular del virus de la peste bovina inactivado por la $\beta$-propiolactona y adyuvado por el adyuvante A 65 no da ninguna protección durante los meses síguiendo su administración en terneros mientras todavia albergan anticuerpos de origen maternal contra la peste. El problema de la vacunación contra la peste bovina de los terneros queda planteado en las campañas de vacunación. 


\section{BIBLIOGRAPHIE}

1. BERGEON et CÉBE. - Au sujet de la vaccination antipestique. Bull. Acod. Vét. Fr., 1930, $3: 358-370$.

2. BÖGEL (K.), ENDERS-RUCKLE (G.) et $\operatorname{PROVOST}\left(A_{\text {. }}\right.$. - - Une réaction sérologique rapide de mesure des anticorps antibovipestiques. C. R. Acad. Sci. (Paris), 1964, 259 : 482-484.

3. BRANAGAN (D.) et HAMMOND (J. A.). Rinderpest in Tanganyika : a review. Bull. epiz. Dis. Afr., 1965, 13 : 225-246.

4. BROWN (R. D.). - Rinderpest immunity in calves. 1. - The acquisition and persistence of maternally derived antibody. 1. Hyg. (Camb.), 1958, 56 : 427-434.

4a. DAUBNEY (R.). Cité par CURASSON (G.). - Traité de Pathologie exotique vétérinaire et comparée. $2^{e}$ édition. Paris, Vigot Frères, éditeurs. 1942.

5. ENDERS-RUCKLE (G.). - Some characteristics of immunity following natural measles and various forms of immunization. Arch. ges. Virusf., 1967, $22: 23-34$.

6. HILLEMAN (M. R.). - Critical appraisal of emulsified oil adjuvants applied to viral vaccines. Prog. Med. Virol., 1966, 8 : 131-182.

7. JACOTOT (H.). - Observations et recherches sur la peste bovine du bétail indochinois. Arch. Inst. Pasteur Indochine, 1932, no 15 : 3-95.

7a. JACOTOT $\left(H_{1}\right)$ et COLSON (M.). - Effets du vaccin contre la peste bovine chez les veaux issus de vaches activement im munisées. Bull. Soc. Path. exot., 1935, 28 : 74-78.

8. Laboratoire de Farcha. - Rapport annuel 1965, 65-67.

9. Laboratoire de Farcha. - Rapport annuel 1966, 104-107.

10. LALANNE (M. A.). - Séro-vaccination et vaccination antipestique en moyenne Côte d'lvoire. Durée de l'immunité. Bull. Serv. zoot. Epiz. A. O. F., 1940, 3 : 167-174.

11. LEPISSIER (H. E.) et MACFARLANE (l.M.). - Campagne conjointe contre la peste bovine, Phase 1, Rapport ferminal novembre 1965. Bull. epiz. Dis. Afr., 1966, 14 : 153-191.
12. LEPISSIER (H. E.) et MACFARLANE (I, M.). - Techniques de vaccination massives en vue de contrôler l'expansion de la peste bovine (Campagne conjointe contre la peste bovine P. C. 15). Bull. O. I. E., 1967, $68: 665-679$.

13. OTT (R. L.), GORHAM (J. R.) et FARRELL (R. K.). - A note on the use of adjuvated distemper vaccine in ferrets. Conad. J. comp. Med., 1965, $28:$ 214-215.

14. POLSON (A.). - Comparative electrophoretic studies of bovine and human colostrum in relation to neo-natal immunity. Ond. $J$. Vet. Res., 1952, $25: 7$.

15. PROVOST (A.), MAURICE (Y.) et BORREDON (C.). - Protection antipestique conférée aux bovins par le virus de la rougeole. Application aux veaux passivement immuns par anticorps maternels. Rev. Elev. Méd. Vét. Poys trop., 1968, $21:$ 145-163.

16. SCOTT (G. R.). - Rinderpest. Adv. Vet. SC., $1964,9: 113-224$.

17. SCOTT (G. R.) et WITCOMB (M. A.).Inactivated rinderpest vaccines, in : EAVRO Report year 1956-57. Government Printer, Nairobi.

18. SMITH (V. W.). - Active immunization of calves with tissue culfured rinderpest vaccine. J. Comp. Path., 1966, $76: 217-224$.

19. STONE (S. S.) and DELAY (P. D.). - The inactivation of rinderpest virus by $\beta$-propiolactone and its effect on homologous complement fixing and neutralizing antibodies. J. Imm., 1961, $87: 464-467$.

20. STRICKLAND (K. L.). - Vaccination of calves against rinderpest. Vet. Rec., 1962, $74: 630-631$.

21. VALLEE, PHILIPPE ef BOURDIE. -- La sérovaccination dans la peste bovine. Bull. Serv. Zoot. Epiz. A. O. F., 1941, $4: 3-9$.

22. WOODHOUR (A. F.), METZGAR (D. P.), STIN (T. B.), TYTELL (A. A.) et HILLEMAN (M. R.). - New metabolizable immunologic adjuvant for human use. I. Development and animal immune response. Proc. Soc. exp. Biol., $1964,116: 516-523$. 